\title{
Ultraschallstreuverfahren zur Charakterisierung von Dispersionen mit hohem Partikelanteil
}

Dipl.-Ing. Sebastian Wöckel, Institut für Automation und Kommunikation (ifak) e.V. Magdeburg, WernerHeisenberg-Str. 1, 39106 Magdeburg, Tel.: +49 391 9901430, sebastian.woeckel@ifak.eu

Dr.-Ing. Ulrike Hempel, Institut für Automation und Kommunikation (ifak) e.V. Magdeburg, WernerHeisenberg-Str. 1, 39106 Magdeburg, Tel.: +49 3919901 427, ulrike.hempel@ifak.eu

Dipl.-Ing. Robert Weser, Technische Universität Dresden - Institut für Verfahrenstechnik und Umwelttechnik, Arbeitsgruppe für Mechanische Verfahrenstechnik, 01062 Dresden, Tel.: +49 35146335226 , robert.weser@tu-dresden.de

Dr.-Ing. Benno Wessely, Technische Universität Dresden - Institut für Verfahrenstechnik und Umwelttechnik, Arbeitsgruppe für Mechanische Verfahrenstechnik, 01062 Dresden, Tel.: +49 35146336321 , benno.wessely@tu-dresden.de

Prof. Dr.-Ing. Jörg Auge, Hochschule Magdeburg-Stendal, IWID - Institut für Elektrotechnik, Postfach 3655, 39011 Magdeburg, Tel.: +49 391 8864388, joerg.auge@hs-magdeburg.de

\section{Motivation}

Eine Reihe von Prozessen der Lebensmittelindustrie sowie verfahrenstechnische oder biotechnologische Anwendungen sind geprägt durch die Charakterisierung der Feststoffphase in hochkonzentrierten Dispersionen. So können die online bzw. inline gewonnenen Daten zur Partikelgrößenverteilung und konzentration dazu beitragen, die verschiedenen Prozesse optimal zu steuern, Produktqualitäten gezielt zu steigern oder Anlagenverfügbarkeiten zu erhöhen. Exemplarische Vorgänge, wie die Emulgierung und (Nass-)Zerkleinerung, finden sich in industriell relevanten Applikatione, $n$ wie der Herstellung von Lacken und Farben oder der Aufbereitung von Erz- und Klärschlämmen. In der Halbleiterfertigung ist das Monitoring von hochgefüllten Säge- und Schleifsuspensionen zur Qualitätssicherung des Herstellungsprozesses ein ebensolches Anwendungsfeld für Partikelmesstechniken.

Eine der etablierten Methoden basiert auf der akustischen Durchschallung der Dispersion und zeichnet sich durch eine schnelle, inline-fähige Arbeitsweise aus, die im Gegensatz zu optischen Methoden auch an hoch konzentrierten, opaken Proben ohne Verdünnung angewendet werden kann. Ferner ist die Ultraschall(US)-Technik auch unter rauen Prozessbedingungen einsetzbar und erlaubt den Einsatz in einem weiten Konzentrations- und Partikelgrößenbereich. Hierzu werden die US-Wandler häufig in einer sich gegenüberliegenden Sender-Empfänger-Anordnung positioniert (vgl. Bild 1) oder ein einzelner, in Sende- und Empfangsbetrieb umschaltbarer Wandler unter Ausnutzung eines Reflektors verwendet. Mit einer solchen Anordnung ist es möglich, sowohl den Transmissions- als auch den Extinktionsanteil (Dämpfung) der mit der Dispersion wechselwirkenden Schallwelle zu bestimmen sowie Informationen zur Dimension, Größenverteilung und Konzentration der Teilchen in Suspensionen und Emulsionen abzuleiten. Aufgrund der erheblichen Schalldämpfung ist es jedoch bei hoch konzentrierten Stoffsystemen erforderlich, den Abstand zwischen Sende- und Empfangswandler hinreichend klein zu gestalten $(0,5 \mathrm{~mm}-2 \mathrm{~mm})$, um ein noch auswertbares Messsignal zu erhalten. Dies führt bei InlineAnwendungen häufig zu Verstopfungen innerhalb der Messstrecke (vor allem beim Vorhandensein einzelner großer Feststoffpartikel oder pastöser Medien), was die Zuverlässigkeit und die Akzeptanz der Messeinrichtung bei den Anwendern einschränkt.

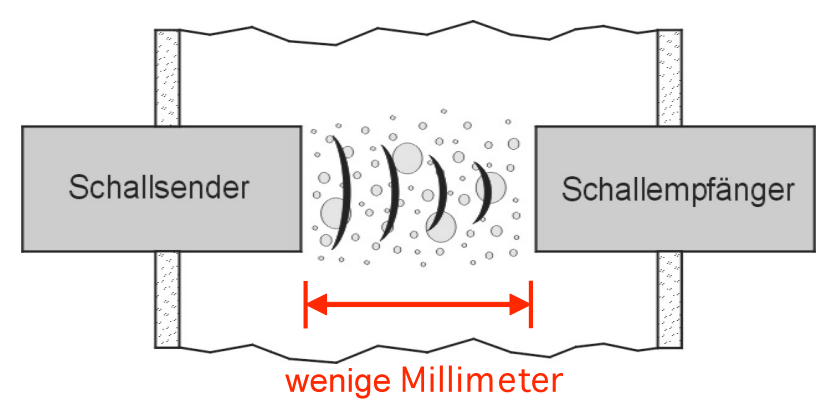

Bild 1: schematischer Aufbau einer US-Transmissionsanordnung 
Der im Beitrag präsentierte Ansatz nutzt zur Partikelcharakterisierung in hochkonzentrierten Dispersionen die vom Stoffsystem gestreuten (statt transmittierten) Schallanteile. Äquivalent zur Extinktionsanordnung ermöglicht dies Aussagen zur dispersen Phase, jedoch ohne das Risiko der kritischen Verstopfung der Messstrecke einzugehen (Vermeidung des Messspaltes). Kerngedanke des experimentellen Aufbaus ist der Einsatz breitbandiger Sende-/Empfangswandler, die hinsichtlich einer hohen Sendeleistung, eines der Eindringtiefe entsprechenden Messfensters und hoher Empfangssensitivität optimal aufeinander abgestimmt sind. Auf diese Weise soll der von den Partikeln gestreute Schallanteil erfasst und hinsichtlich der Intensität, der Laufzeit (entspricht der Eindringtiefe) und der Schallfrequenz analysiert werden. Neben dem klassischen Messverfahren der US-Dämpfungsspektroskopie (Extinktionsprinzip) existieren bereits Messverfahren bzw. prozessfähige Messgeräte, die auf der Analyse der Schallstreuung an einzelnen Partikeln beruhen [UPM Ultrasonic Particle Monitor, Netherlands Organization for Applied Scientific Research TNO, http://www.tpd.tno.nl], [Model AS3 Acoustic Sensor, Galvanic Applied Sciences Inc., http://www.galvanic.com]. Den gemessenen Streusignalen werden dabei sogenannte Einzelechos, d. h., von einzelnen Partikeln reflektierte Schallwellen zugeschrieben. Diese Messgeräte sind für den Einsatz in sehr gering konzentrierten Partikelsystemen konzipiert, da sie einzelne und voneinander unabhängige Streuereignisse (Einfachstreuung) auswerten und meist zur Einzelpartikeldetektion genutzt werden. Somit sind derartige Messverfahren nicht in der Lage, Streusignale aus hochkonzentrierten Dispersionen zu interpretieren.

\section{Messaufbau}

Bild 2 verdeutlicht schematisch den grundlegenden Aufbau des auf Ultraschallstreuung basierenden Verfahrens. Im Hinblick auf eine kostenoptimierte Lösung wird kommerziell verfügbare Hardware der Neuentwicklung einer Spezialhardware vorgezogen. Bei der Anordnung der US-Wandler werden mehrere Konzepte verfolgt. Neben der klassischen Reflexion (ein Sende-/Empfangswandler mit Vorlaufstrecke, direkte Rückstreuung) sollen auch die unter definierten Winkeln gestreuten Schallanteile (getrenntes USWandlerpaar, winkelabhängige Streuung, Bild 3) analysiert werden. Die Bereitstellung definierter Messbedingungen ist für die experimentellen Untersuchungen von besonderer Bedeutung. So wird die Dispersion während der Messung temperiert und der Dispergierzustand sowie die Partikelkonzentration mittels Laboranalytik (Partikelgrößenmessung mit Laserbeugungsspektrometer, Partikelkonzentrationsmessung mit Verdampfungswaage) überwacht. Weiterhin muss durch eine geeignete Strömungsführung vor der US-Messsonde sichergestellt werden, dass das Partikelsystem homogen sowie zufällig veränderlich im Messvolumen verteilt ist.

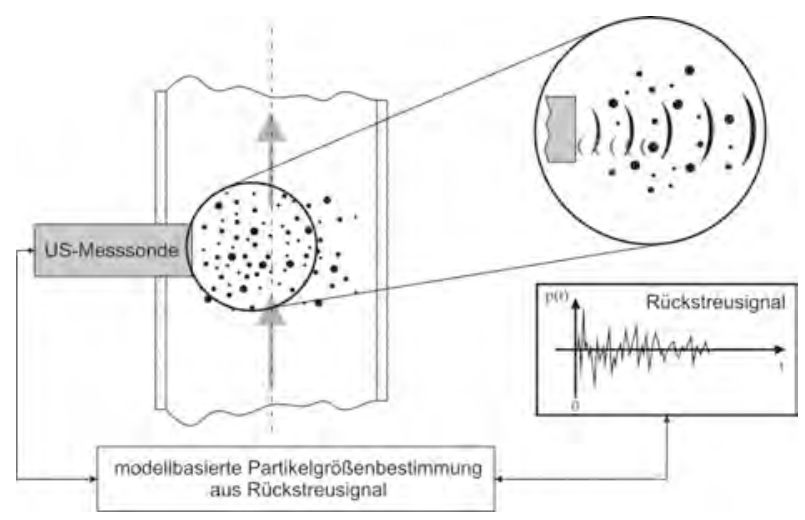

Bild 2: Ultraschallrückstreuung

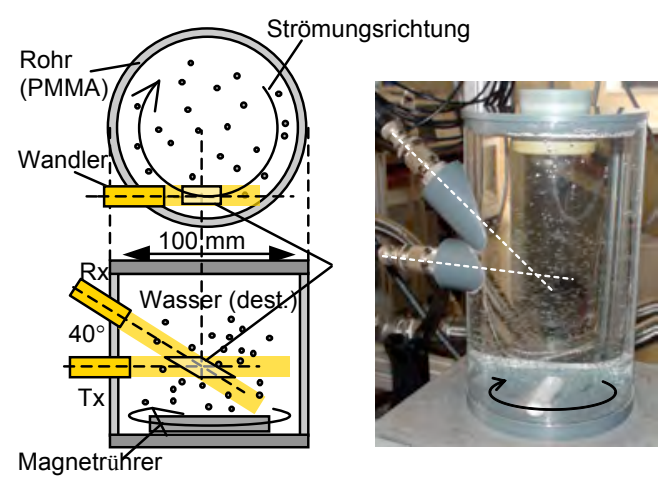

Bild 3: Messvolumen für winkelabhängige Ultraschallstreuung: Schematische Darstellung (links) und Foto des Messaufbaus mit Polymerpartikeln in Wasser

\section{Direkte Rückstreuung}

Der in Bild 2 schematisch gezeigte Ansatz unter Nutzung des direkt zurückgestreuten Echosignals wurde mit einem in Sende-/Empfangsmodus umschaltbaren Wandler (Mittenfrequenz: $4,5 \mathrm{MHz},-6 \mathrm{~dB}-$ Bandbreite: $3 \mathrm{MHz}$, Fa. Olympus) und konventioneller Impulsanregung (US-Pulser/Receiver UT340 der Fa. Utex Scientific Instruments, Inc.) umgesetzt. Den detaillierten Aufbau der Messanordnung zeigt Bild 4. Um die aktive Oberfläche der Tauchwandler vor Abrasion durch die Partikel zu schützen, wurde eine wasserbefüllte Vorlaufstrecke eingesetzt (Länge: $20 \mathrm{~mm}$ ). Die Trennung zwischen Vorlaufstrecke 
und Partikelsystem wurde durch den Einsatz eines akustischen Fensters erreicht. Der US-Wandler nebst kompletter Vorlaufstrecke wurde als Einstecksonde konzipiert. Auf diese Weise lässt sich ein einfacher Messaufbau realisieren, wobei die Einstecksonde für die Messung in eine gerührte Suspension getaucht wird. Das Rühren sorgt neben einer guten Durchmischung der Partikel ebenfalls dafür, dass die Partikel innerhalb der Messzone (vor dem Fenster) möglichst gleich verteilt sind.

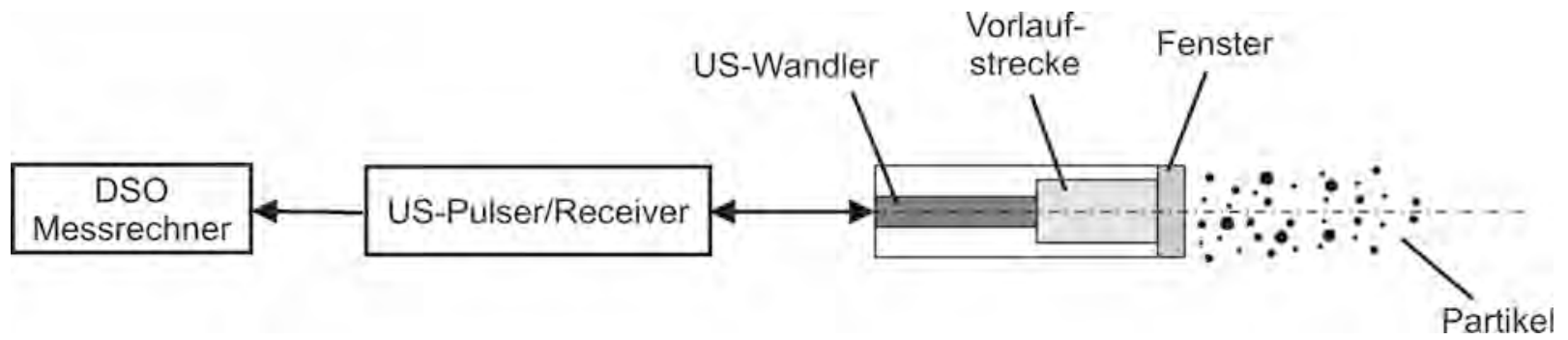

Bild 4: Messaufbau zur Erfassung von Ultraschallrückstreusignalen (das vom US-Receiver ausgegebene Signal wird mittels eines Digitalspeicheroszilloskops (DSO) erfasst und im Messrechner weiterverarbeitet)

Die direkte Ultraschallrückstreumessung liefert nach Anregung des Schallwandlers ein Echosignal (Amplitude $p$ in Abhängigkeit von der Laufzeit $t$ ), das neben den Reflexionspeaks des Vorlaufstreckenfensters ebenfalls die Rückstreusignale der Partikel enthält. Ein solcher Signalverlauf ist in Bild 5 gezeigt.

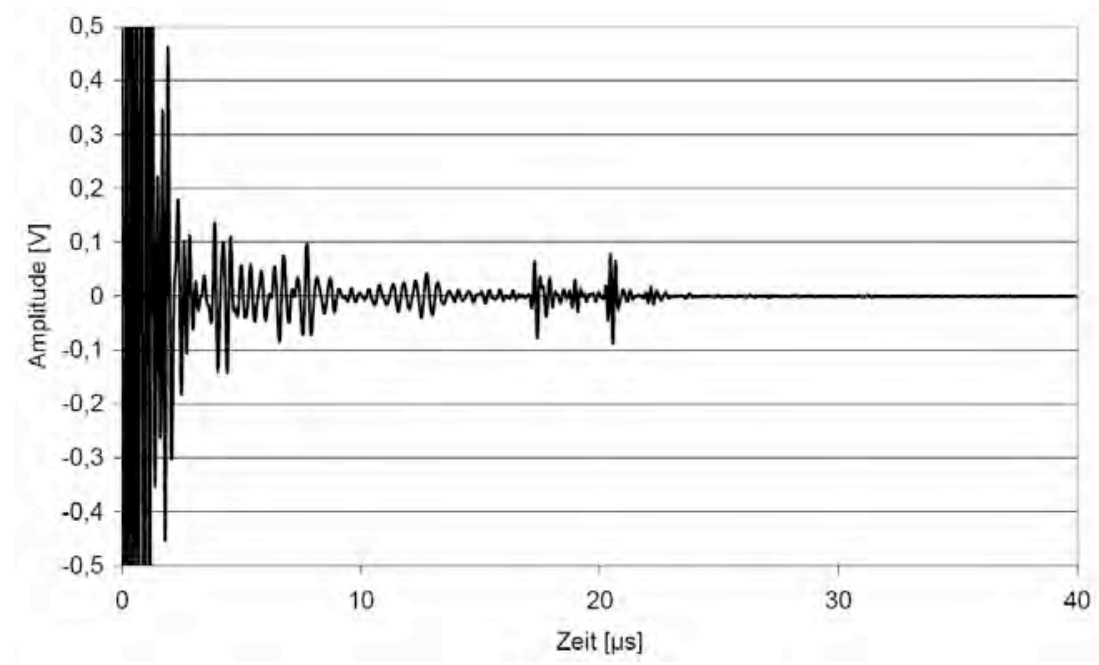

Bild 5: US-Rückstreusignal (dargestellt als äquivalente elektr. Spannung über der Laufzeit), Fensterreflexionen (zu Beginn) und zugehörige Mehrfachreflexionen dominieren den Signalverlauf

Aus dem Echo- bzw. Rückstreusignal wird deutlich, dass die durch Partikel erzeugten Signalkomponenten aufgrund ihrer geringen Amplitude nur unzureichend von Signalanteilen infolge der (Mehrfach-)Reflexion im Fenster zu unterscheiden sind. Daher wurde anstelle der absoluten Auswertung der Echoamplitude deren Standardabweichung in Abhängigkeit von der Laufzeit herangezogen. Nach Erfassung von $N$ Einzelmessungen (vollständiges Echosignal infolge eines ausgesendeten Impulses) wird zu jeder Laufzeit $t$ die Standardabweichung der jeweils $N$ Amplitudenwerte bestimmt. In der Folge stellen die Fenster- und deren Mehrfachreflexionen statische Signalanteile im gesamten Echosignal dar und liefern somit keinen Anteil zur Standardabweichung. Demgegenüber erzeugen die zeitlich veränderlichen Partikelsignale (infolge der Bewegung der Partikel durch das Messvolumen) eine deutlich messbare Standardabweichung (Bild 6). 


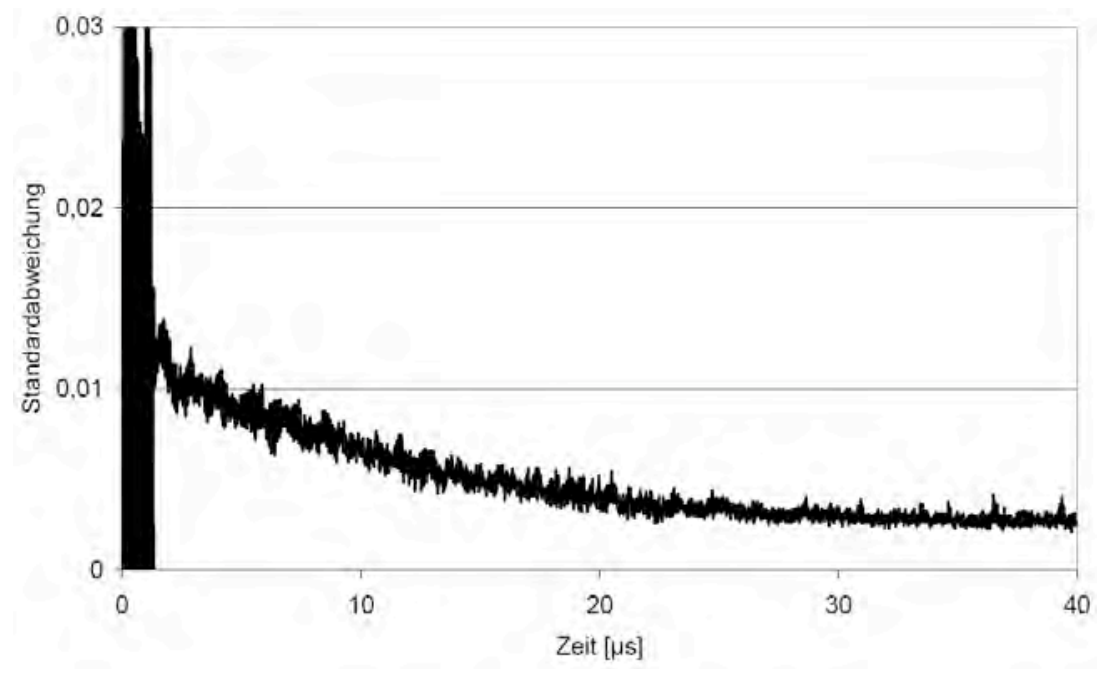

Bild 6: Über $N=100$ Einzelmessungen ermittelte laufzeitabhängige Standardabweichung des Ultraschallechosignals aus einer Dispersion

Beim Durchlaufen der Dispersion werden sowohl die emittierte als auch die an den Partikeln gestreuten Schallwellen in Abhängigkeit von der zurückgelegten Wegstrecke gedämpft. Die vom Schallwandler detektierbare Echosignalamplitude verringert sich demnach mit zunehmender Laufzeit - gleiches gilt für die Standardabweichung. Die Schallamplitude fällt dabei exponentiell mit der durchschallten Wegstrecke bzw. der Laufzeit ab und wird u. a. von der Partikelgröße und -konzentration bestimmt [1], [2]. Dieser Effekt konnte in einer exemplarischen Messreihe nachvollzogen werden. Dazu wurde die direkte USRückstreuung aus einer Suspension von $\mathrm{SiO}_{2}$-Partikeln (Dichte: $2,35 \mathrm{~g} / \mathrm{cm}^{3}$, mittlere Partikelgröße: $3 \mu \mathrm{m}$, s. Bild 7) in Wasser aufgenommen.

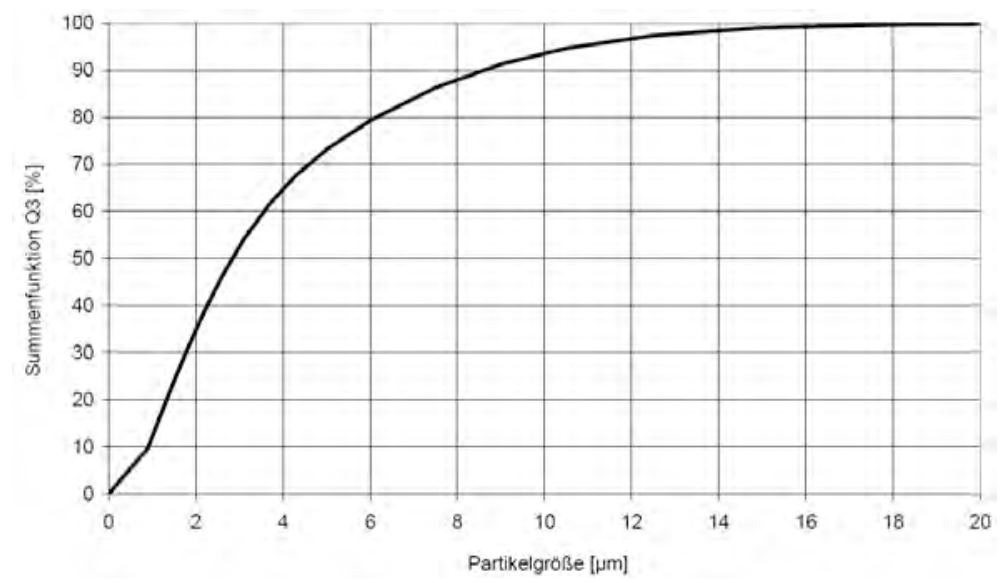

Bild 7: Partikelgrößenverteilung (Summenfunktion) der verwendeten $\mathrm{SiO}_{2}$-Partikel

Die mit dem US-Wandler $(4,5 \mathrm{MHz})$ emittierten Schallwellen haben in Wasser, wie auch in der Suspension, eine Wellenlänge von ca. $330 \mu \mathrm{m}$. Die Partikelgröße liegt demnach eine Größenordnung unterhalb der Wellenlänge - entsprechend schwach ausgeprägt ist die Schallstreuung durch die Partikel [2]. Die nachfolgend beschriebenen Ergebnisse einer Konzentrationsmessreihe zeigen, dass selbst unter diesen Voraussetzungen die messtechnische Erfassung und Auswertung von US-Reflexions- bzw. Rückstreusignalen möglich ist.

Im Rahmen der Messreihe wurde dazu die Partikelkonzentration stufenweise auf bis zu 50 Ma.-\% erhöht und die entsprechenden Suspensionsansätze zusätzlich mit einem US-Spektrometer [3] vermessen. Im Ergebnis der Messungen zeigt Bild 8 (links) den zeitlichen Verlauf der Standardabweichung für die verschiedenen Partikelkonzentrationen. Die Standardabweichung ist logarithmiert (in Dezibel) aufgetragen, da der exponentielle Einfluss der Schalldämpfung auf diese Weise linearisiert dargestellt werden kann. Mit zunehmender Partikelkonzentration fallen die an die Kurvenverläufe angepassten 
Geraden (Fit-Funktionen, vgl. Bild 8 rechts) stärker ab, gleichzeitig steigt die Schalldämpfung an. Der Betrag der Geradenanstiege entspricht der (laufzeitabhängigen) Schalldämpfung, z. B. 0,5 dB/ $\mu$ s bei 10 Ma.-\%.
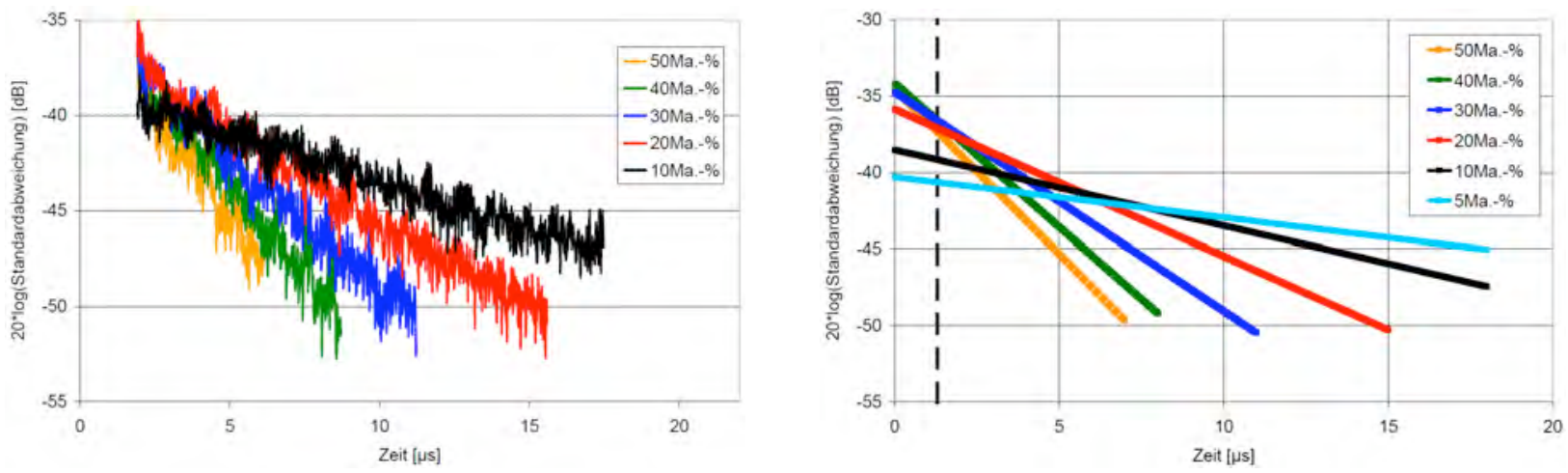

Bild 8: konzentrationsabhängige Standardabweichung in logarithmischer Darstellung (links) und Fit der Signalverläufe (rechts), gestrichelte Linie entspricht der Fensterposition

Die mit dieser Methode bestimmte Schalldämpfung korreliert mit den Werten der Referenzmessung (Tab. 1). Für eine bessere Vergleichbarkeit wurden die laufzeitabhängigen Dämpfungswerte (Einheit $\mathrm{dB} / \mu \mathrm{s}$, fett gedruckt) unter Verwendung der Schallgeschwindigkeit $(1450 \ldots 1500 \mathrm{~m} / \mathrm{s}$, abhängig von der Partikelkonzentration) in wegstreckenabhängige Werte umgerechnet. Da die Dämpfungswerte des USSpektrometers zudem auf die jeweilige Frequenz bezogen sind, wurde zudem eine Normierung der Messwerte auf die Mittenfrequenz des US-Wandlers (4,5 MHz) durchgeführt.

Tabelle 1: konzentrationsabhängige US-Dämpfung aus Rückstreumessungen, vergleichende Ergebnisse aus Referenzmessung mit US-Spektrometer DT1200 (Dispersion Technology, Inc.)

\begin{tabular}{|c|c|c|c|}
\hline $\begin{array}{c}\text { Feststoffanteil } \\
\mathrm{SiO}_{2}[\mathrm{Ma} .-\%]\end{array}$ & $\begin{array}{c}\text { Dämpfung aus Rückstreusignal } \\
{[\mathrm{dB} / \mathrm{\mu s}]}\end{array}$ & $\begin{array}{c}\text { Dämpfung aus Rückstreusignal } \\
{[\mathrm{dB} / \mathrm{cm} / \mathrm{MHz}]}\end{array}$ & $\begin{array}{c}\text { Dämpfung US-Spektrometer } \\
\text { (DT1200) [dB/cm/MHz] }\end{array}$ \\
\hline 10 & $\mathbf{0 , 5}$ & 0,75 & 0,63 \\
\hline 20 & $\mathbf{0 , 9 6}$ & 1,44 & 1,36 \\
\hline 30 & $\mathbf{1 , 4 3}$ & 2,16 & 2,12 \\
\hline 40 & $\mathbf{1 , 9}$ & 2,89 & 3,84 \\
\hline 50 & $\mathbf{2 , 1 6}$ & 3,31 & 3,48 \\
\hline
\end{tabular}

Bleibt, wie im Fall der gezeigten Messergebnisse, die Partikelgröße konstant, so kann die auftretende Änderung der Schalldämpfung demnach dazu genutzt werden, um eine Änderung der Partikelkonzentration zu erkennen.

In vielen Anwendungsfällen ist die Durchführung dieser einfachen Korrelation jedoch nicht möglich bzw. nicht sinnvoll, da sich neben der Konzentration oftmals auch die Partikelgröße ändert. In weiteren Arbeiten soll daher neben der Schalldämpfung die Rückstreuamplitude als zusätzlicher Parameter aus den Rückstreusignalen extrahiert und für die Auswertung genutzt werden. Bei genauerer Betrachtung der Darstellung in Bild 8 (rechts) ist zu erkennen, dass die Fitgeraden auf Höhe der Fensterposition unterschiedliche Funktionswerte aufweisen: je nach Partikelkonzentration weist die Rückstreuamplitude (bzw. deren Standardabweichung) einen anderen Betrag auf. Frühere Untersuchungen zeigen zudem, dass die (zurück)gestreute Schallamplitude neben der Konzentration auch von der Partikelgröße abhängig ist [7]. Mit der Rückstreuamplitude steht demnach ein messbarer Parameter zur Verfügung, der in Verbindung mit der Schalldämpfung, eine qualifiziertere Aussage über das Partikelsystem zulässt. 


\section{Winkelabhängige Streuung}

Die Verwendung eines Wandlers im Impuls-Echo-Verfahren führt dazu, dass durch die Umschaltung nur zeitlich begrenzte transiente Signale verwendet werden können, die Empfindlichkeit des Empfangswandlers nicht anpassbar ist und für irreguläre Partikel keine winkelabhängige Streuinformation vorliegt. Entsprechend schließen die Untersuchungen die winkelabhängige Streuung mit ein. Entscheidende Vorteile sind die Trennung von Sender und Empfänger zur Gewährleistung einer hohen Empfindlichkeit sowie die Möglichkeit, kontinuierliche oder längere Signalsequenzen zu verwenden.

Die Schallstreuung an Einzelpartikeln, d.h. die messbare reflektierte Leistung $P_{\text {OUT }}(1)$, ist abhängig vom Material der Partikel (Reflektivität des Partikels, $\Gamma$ ) und deren Geometrie (winkelabhängige Streufunktion, $\sigma$ ) in Relation zur eingesetzten Anregungsfrequenz $f$. Bild 9a zeigt hierzu ein vereinfachtes Modell der winkelabhängigen Schallstreuung an Einzelpartikeln mit dem sich GI. 1 zur Bewertung der Leistungsfähigkeit des Streumessverfahrens ableiten lässt.

Ein bewegtes Partikel erzeugt im Sensitivitätsbereich der Schallwandler (Bild 9b) ein in Bild 9c dargestelltes Streusignal, an dem sich die Geschwindigkeit anhand der Geradensteigung und die Streuleistung $P_{\text {OUT }}$ des Partikels anhand der maximalen Amplitude experimentell bestimmen lassen. Dargestellt sind die Einhüllenden von aufeinanderfolgenden Einzelmessungen im Abstand von $20 \mu s$.

$$
\frac{P_{O U T}}{P_{I N}}=\frac{4 k_{P}}{\pi d_{W}^{2}} \cdot \frac{\pi d_{P}^{2}}{4} \cdot \sigma \cdot \Gamma \cdot \frac{1}{4 \pi s^{2}} \cdot \frac{\pi d_{W}^{2}}{4} k_{P}
$$
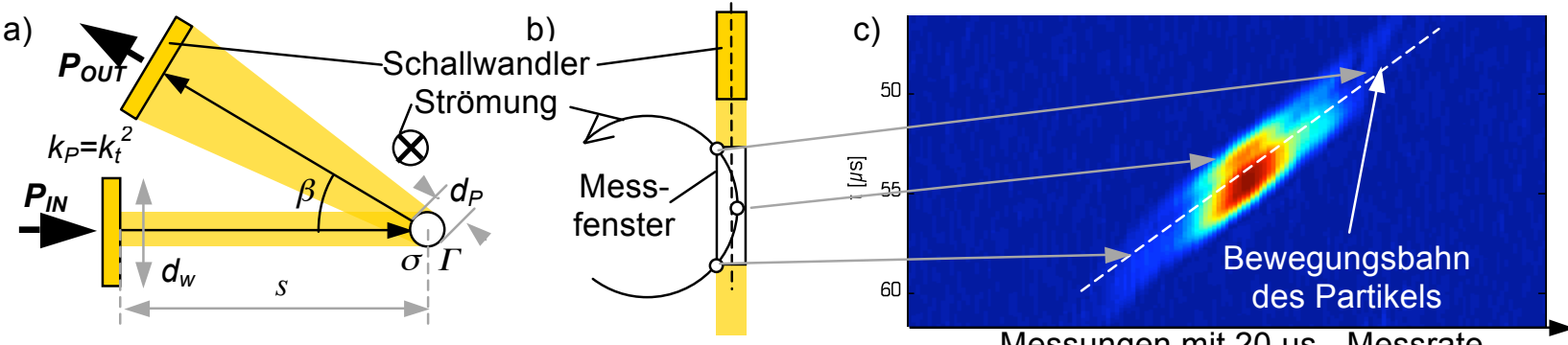

Bild 9: a) Schematische Darstellung der Streumessung ( $\Gamma$ - Reflektivität des Partikels, $\sigma-$ winkelabhängige Streufunktion, $k_{P}-$ Koppelfaktor, $d$-Durchmesser, $s$ - Weg), b) Sicht auf Wandler, Messfenster [15 $\mu$ s] und Partikelbahn, c) Streusignal eines sich durch das Messfenster bewegenden Partikels $d=200 \mu \mathrm{m}$ nach Messaufbau Bild 3

Entsprechend Gleichung 1 ergibt sich unter idealisierten Bedingungen für ein Polymerpartikel mit $d_{P}=$ $200 \mu \mathrm{m}$, einer effektiven Wandlerfläche $d_{w}=6 \mathrm{~mm}$, einem Koppelfaktor $k_{P}=0,02$ und einem Streuabstand $s=45 \mathrm{~mm}$ eine maximal theoretisch messbare Streuamplitude von $U_{p p}=0,85 \mathrm{mV}$. Der Messaufbau nach Bild 3 liefert eine Amplitude von $U_{p p}=0,6 \mathrm{mV}$, welche unter Berücksichtigung der Einfachheit des Modells (1) für weitere Untersuchungen an hochdispersen Systemen eine verwertbare Aussage zur real messbaren Streuleistung liefert.

\section{Äquivalente Signalkodierung}

Mit zunehmender Partikelanzahl in höher konzentrierten Dispersionen nimmt die Mehrfachstreuung zu und verringert das messbare Einzelstreusignal nach GI. 1 zusätzlich. Die Eindringtiefe verringert sich gleichbedeutend. Folglich muss zur Erfassung der Streuung mit repräsentativer Eindringtiefe in das Messvolumen eine höhere Schallleistung $P_{I N}$ in die Suspension eingebracht werden. Für eine hohe Ortsauflösung ist ferner ein Impuls kurzer Zeitdauer $T_{0}$ notwendig. Begrenzt wird die Forderung nach höherer Schallleistung durch die energetische Belastungsgrenze von piezoelektrischen Wandlern und die Linearität des Mediums in Abhängigkeit der Druckamplitude.

Alternativ zu den üblicherweise verwendeten Kurzzeitimpulsen ist insbesondere zur Erhöhung der Leistung die Verwendung von kodierten Signalfolgen möglich [4]. Mit diesen lässt sich die Energie des Sendesignals auf das gesamte / ein größeres zeitliche(s) Messfenster $T$ verteilen. Hierdurch verringert sich das Verhältnis von Maximalamplitude zu Effektivwert (Crest-Faktor) deutlich und ermöglicht die Verwendung geringerer elektrischer Spannungen $U_{T}$ bzw. Druckamplituden im Medium. Das Frequenzspektrum und die Korrelation der Streusignale für Impulsanregung und kodierte Signalfolge sind identisch und stellen die Impulsantwort des Partikelsystems dar [5]. Am Beispiel einer Streumessung unter $90^{\circ}$ mit getrennten Sende- und Empfangswandlern sind in Bild 10 die Korrelationssignale $U_{M}$ von 
bewegten Einzelpartikeln $(500-750 \mu \mathrm{m})$ aus Polymer für einen Impuls mit $U_{0}=55 V_{\text {pp }}$ und eine Signalfolge mit $U_{T}=7,8 \mathrm{~V}_{\mathrm{pp}}$ Anregungsamplitude dargestellt. Äquivalent lässt sich im Korrelationsraum eine effektive Streudämpfung anhand der Standardabweichung oder Amplitude ableiten (s.o.). Voraussetzung zur Verwendung dieses Ansatzes ist ein lineares System, dessen Materialeigenschaften nicht von der Amplitude des eingeprägten akustischen Druckfeldes abhängen.

a) $90^{\circ}$-Streuung bei Impulsanregung

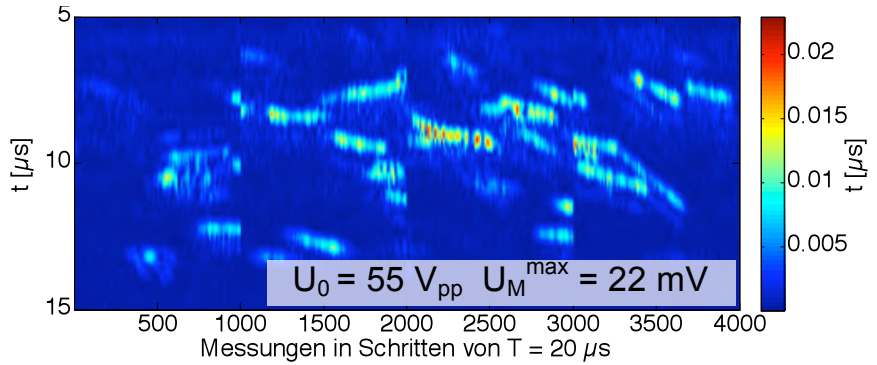

b) $90^{\circ}$-Streuung mit äquivalenter Signalfolge

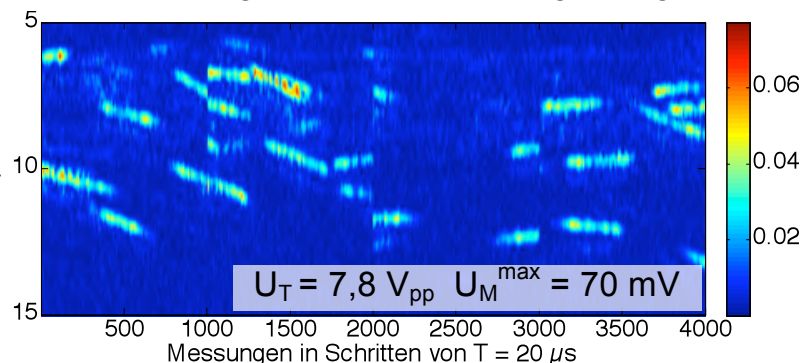

Bild 10: Einhüllende der Einzelstreuungen $U_{M}$ für $\beta=90^{\circ}$ eines 25-MHz-Breitbandsignals an strömenden Polymerpartikeln (500-750 $\mu \mathrm{m})$ in Wasser für verschiedene Anregungssignale gleicher Leistung. a) Impuls; b) kodierte Signalfolge.

\section{Parameter Standardabweichung}

In Anlehnung an Darstellung 8 lassen sich mit den kodierten Folgen Standardabweichungen $\sigma$ bestimmen, welche von der Partikelkonzentration abhängen. Ein Vergleich (Bild 11) der Standardabweichungen eines Partikelsystems CP3000 mit Partikelgrößen von $x_{50}=12 \mu \mathrm{m}$ bei Verwendung einer Impulsanregung und eines kodierten Signals mit Mittenfrequenz von $f=5 \mathrm{MHz}$ für $0^{\circ}$ Reflexionsmessungen zeigt, dass beide Messverfahren äquivalente Aussagen zur maximalen Standardabweichung $\sigma_{\max }$ und deren Abfall $\Delta \sigma$ in Abhängigkeit der Konzentration liefern.

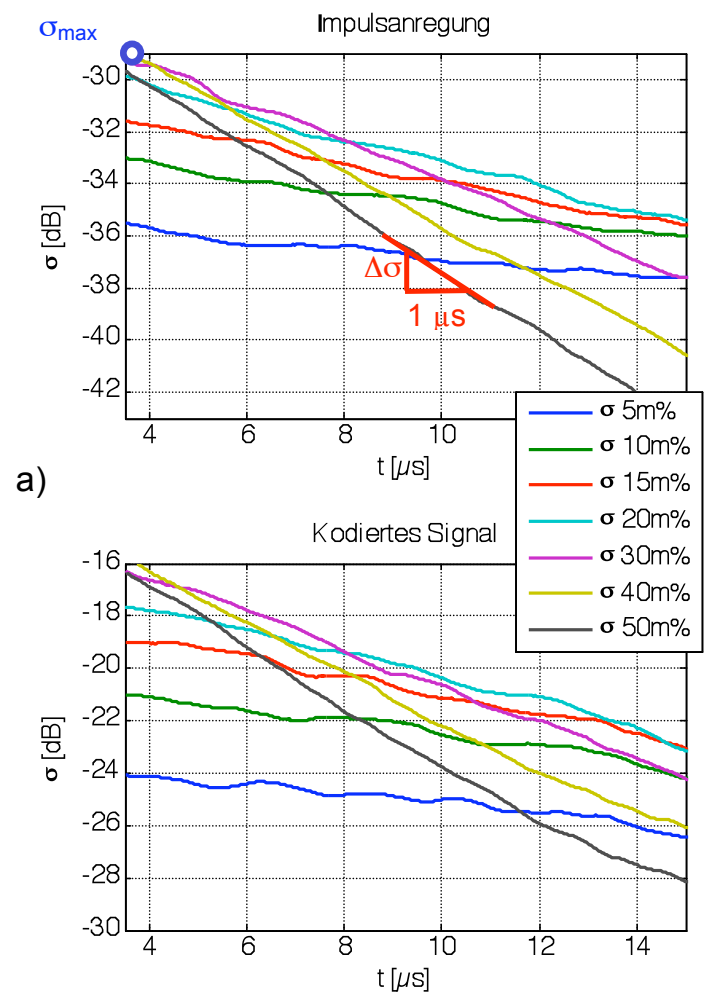

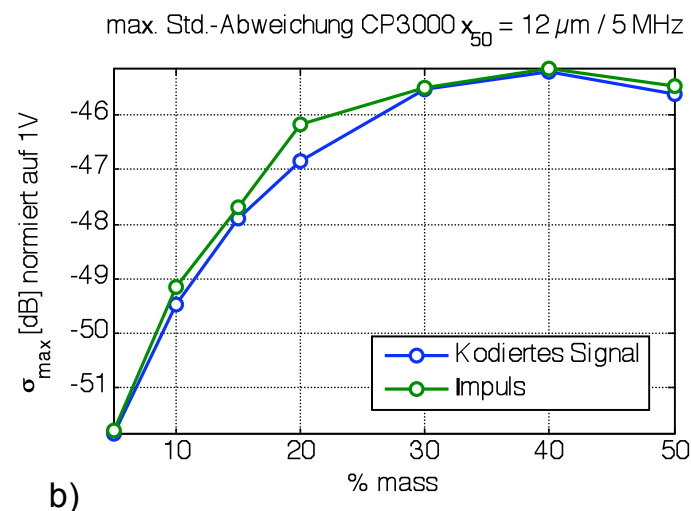

b)

mittlerer Anstieg CP3000 $\mathrm{x}_{50}=12 \mu \mathrm{m} / 5 \mathrm{MHz}$

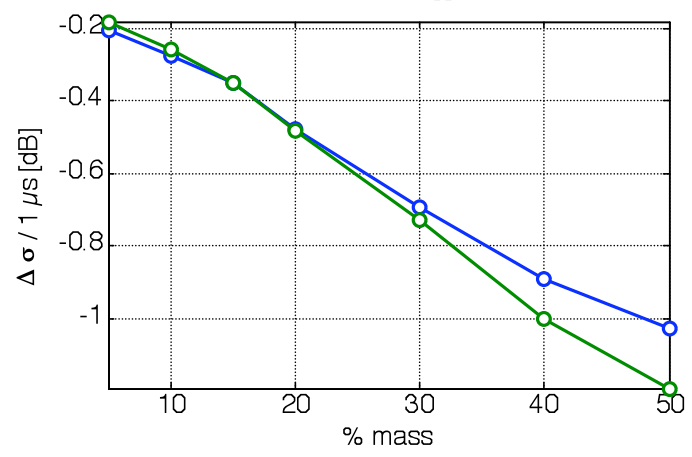

Bild 11: a) Standardabweichung $\sigma\left(U_{M}\right)$ des $0^{\circ}$-Reflexionssignals (Mittenfrequenz $f=5 \mathrm{MHz}$ ) in Abhängigkeit der Eindringtiefe (Zeit t) an einem Glaspartikelsystem (CP3000 $x_{50}=12 \mu \mathrm{m}$ ), ermittelt mit Impulsanregung und äquivalentem kodierten Signal für verschiedene Massenkonzentrationen [5...50 m\%]; b) Parameter der maximalen und differentiellen Standardabweichung normiert auf [1V] Referenzamplitude für Impulsanregung und äquivalentem kodierten Signal im Vergleich. 


\section{Zusammenfassung}

Die Auswertung der laufzeitabhängigen Standardabweichung erlaubt die Bestimmung der US-Dämpfung ohne limitierenden Messspalt, wobei die experimentell in Beispielstoffsystemen ermittelten Werte mit jenen des US-Spektrometers gut übereinstimmen. Aus der Reflexionsmessung lassen sich zwei unabhängige Parameter $\sigma_{\max }$ und $\Delta \sigma$ ableiten. Eine spektrale Analyse der gestreuten Schallsignale steht im Fokus zukünftiger Arbeiten. Die Verteilung der Energie auf das gesamte Messfenster durch Verwendung kodierter Signale begünstigt zudem die Streumessung in Suspensionen mit hoher Partikeldichte (>50 Ma.-\%) und Medien hoher Dämpfung [6]. Die eingebrachte Schalleistung bei konstanter Spannungsamplitude ist um den Faktor des Tastverhältnisses $T / T_{0}$ höher als bei einem Kurzzeitimpuls äquivalenter Bandbreite und Ortsauflösung.

\section{Förderung}

Das IGF-Vorhaben 16681 BR/2 der Forschungsvereinigung Forschungsgesellschaft für Messtechnik, Sensorik und Medizintechnik e.V. Dresden - fms, Theodor-Heuss-Allee 25, 60486 Frankfurt am Main wurde über die AiF im Rahmen des Programms zur Förderung der industriellen Gemeinschaftsforschung und -entwicklung (IGF) vom Bundesministerium für Wirtschaft und Technologie aufgrund eines Beschlusses des Deutschen Bundestages gefördert.

Für die Entwicklung des Messaufbaus und die Erstellung der umfangreichen Messdaten zur Partikelstreuung danken wir Herrn Dipl.-Ing. H. Arndt (ifak).

\section{Literatur}

[1] R. Millner: Wissensspeicher Ultraschalltechnik. 1. Aufl. Leipzig: VEB Fachbuchverlag 1987.

[2] A.S. Dukhin, P.J. Goetz: Ultrasound for Characterizing Colloids, Elsevier, 2002.

[3] DT100 / DT1200 Ultraschallspektrometer, Dispersion Technology, Inc., http://dispersion.com

[4] H. Alrutz: Über die Anwendung von Pseudorauschfolgen zur Messung an linearen Übertragungssystemen. Dissertation, Georg-August-Universität Göttingen, 1983.

[5] J. Sachs, R. Thomä: Vergleichende Untersuchungen zum Einsatz ausgewählter Testsignale in der akustischen Materialprüfung, DAGA 1996, Saarbrücken, 1996.

[6] A. Nowicki, I. Secomski, J.L. Trots: Extending penetration depth using coded ultrasonography. Bulletin of the polish Academy of Sciences. Band 52, Nr. 3, 2004.

[7] J.J. Faran: Sound Scattering by Solid Cylinders and Spheres. J. Acoust. Soc. Am. 23 (1951), 405-418 